\title{
Negative extrathoracic pressure in treatment of respiratory failure in infants and young children
}

\author{
M P Samuels, D P Southall
}

\author{
Abstract \\ Objective-To assess the efficacy of a newly \\ developed system for applying continuous or inter- \\ mittent negative (subatmospheric) extrathoracic \\ pressure in respiratory failure.
}

Design-Uncontrolled clinical trials in infants deteriorating or failing to improve despite standard medical treatment.

Setting-Paediatric and neonatal intensive care units and paediatric wards.

Patients -88 Infants and young children aged 1 day to 2 years with respiratory failure due to bronchopulmonary dysplasia, the neonatal respiratory distress syndrome, bronchiolitis, myopathy, the congenital hypoventilation syndrome, pneumonitis, and postoperative phrenic nerve palsy. At the start of treatment 59 were receiving $\geqslant 50 \%$ inspired oxygen and 40 positive airway pressure ventilation.

Intervention-Treatment was provided within purpose built Perspex chambers of appropriate size. The chamber incorporated safe and effective latex neck seals; facilities for access, monitoring, and observation; and a heater to control the ambient air temperature.

Main outcome measures - Inspired oxygen concentration and carbon dioxide pressure before application of negative extrathoracic pressure and two and $\mathbf{4 8}$ hours afterwards; duration of treatment; and final outcome (discharge home or death).

Results-While arterial oxygen saturation was maintained at constant values 75 infants showed reductions in inspired oxygen concentrations (range 4-50\%, median 15\%) two hours after starting treatment and 74 showed reductions at 48 hours $(2-79 \%$, median 20\%). Of 59 infants who had carbon dioxide pressure measured before and after starting negative extrathoracic pressure, 21 showed a reduction (range $0.6-8.9 \mathrm{kPa}$, median 2.0$), 30$ no change $( \pm 0.5$ $\mathrm{kPa}$ ), and eight a rise (range $0 \cdot 6-5 \cdot 1 \mathrm{kPa}$, median $2 \cdot 1$ ). In 28 patients extubation was facilitated, 54 patients were discharged home, where six continued treatment, and 34 died. Treatments lasted for between two and 236 days (median 13 days).

Conclusion-Negative pressure respiratory support is a non-invasive yet effective treatment for respiratory failure. It may avoid the need for intubation, reduce the pathophysiological consequences of positive airway pressure ventilation, and aid extubation.

Department of Paediatrics, National Heart and Lung Institute, Brompton Hospital, London SW3 6HP

M P Samuels, MRCP, paediatric research fellow D P Southall, MD, consultant paediatrician

Correspondence to: $\mathrm{Dr}$ Samuels.

BrMed f 1989;299:1253-7 infection. In addition, positive airway pressure ventilation produces barotrauma, has adverse haemodynamic effects, and may contribute to the develop- ment of chronic lung disease, particularly in preterm infants. It is mostly practised in intensive care units and requires the use of complicated equipment by highly trained nursing and medical staff.

Subatmospheric (negative) extrathoracic pressure as a means of respiratory support has a long history. ${ }^{12}$ It was used to treat children with respiratory failure due to poliomyelitis and in the 1960 s and 'seventies was found to be effective in the management of the neonatal respiratory distress syndrome. ${ }^{3-10}$ More recently it has been used in persistent pulmonary hypertension of the newborn $^{11}$ and pulmonary interstitial emphysema. ${ }^{12}$ Its value, however, has been hindered by technical problems, ${ }^{9}$ which have included upper airway obstruction and soreness from the neck seal and difficulties in achieving access to the patient and maintaining a neutral thermal environment for newborn infants. These problems have been overcome by the development of a new system for applying negative pressure respiratory support. We present the results of the system's use in uncontrolled trials during which it was developed.

Patients, materials, and methods

Table I shows the clinical details of 83 patients treated with continuous negative extrathoracic pressure and of five who received intermittent negative pressure ventilation. In all of them their clinical condition had deteriorated despite standard treatment.

TABLE I-Causes of respiratory failure and ages of patients treated with negative extrathoracic pressure

\begin{tabular}{lrll}
\hline Diagnosis & \multicolumn{1}{c}{$\begin{array}{c}\text { No of } \\
\text { patients }\end{array}$} & \multicolumn{1}{c}{ Age range } \\
\hline Bronchopulmonary dysplasia & 47 & $4-38$ weeks \\
Neonatal respiratory distress syndrome & 13 & $1-12$ days \\
Phrenic nerve palsy (postoperative) & 10 & 12 days-2 years \\
Bronchiolitis or asthma & 7 & $2-12$ months \\
Myopathy & 5 & $5-17$ months \\
Complications of cardiac surgery & 2 & 6 and 12 weeks \\
Pneumonitis & 2 & 17 days and 5 months \\
Congenital alveolar hypoventilation syndrome & 2 & Both 3 weeks \\
\hline Total & 88 & 1 day-2 years
\end{tabular}

Before starting treatment 40 patients were receiving positive airway pressure ventilation and 59 required $\geqslant 50 \%$ inspired oxygen to maintain adequate arterial oxygen saturation (defined below), 35 of them requiring 95-100\% inspired oxygen. Continuous negative extrathoracic pressure was used to avoid intubation in acute respiratory failure, to reduce additional inspired oxygen concentrations and positive airway pressure in infants already intubated, and to help in weaning infants from positive airway pressure ventilation. Two infants with bilateral phrenic nerve palsies (after cardiac surgery), one with myopathy, and two with the congenital alveolar hypoventilation syndrome were treated with long term intermittent negative extrathoracic pressure ventilation to continue respiratory support after extubation. Treatment was started in this hospital (38 patients) or in the hospital of referral ( 50 patients). 


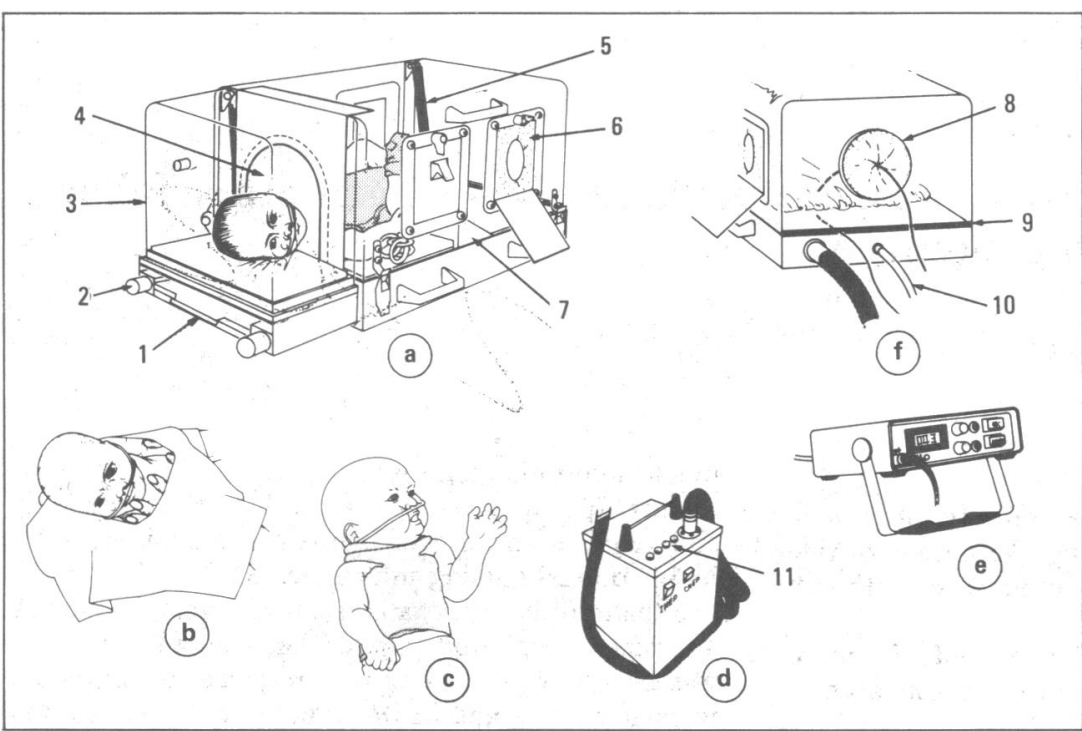

FIG 1-System for applying negative extrathoracic pressure. (a) Negative pressure chamber with head box (b) latex neck seal being placed over infant's head, (c) stockinette neck protector, (d) electrical fan unit with facility for continuous or intermittent suction, (e) pressure monitor, ( $f$ foot end of chamber showing access for monitoring leads, infusion lines, and suction hose. Details of the chamber include release for head section ( 1 ), rods on which it slides out away from chamber base (2), head box (3), latex neck seal taped up over arch in lid (4), gas strut hinges (5), foam gasket in porthole (6), footplate to support infant when chamber is tilted up (7), porthole for infusions, etc (8), rubber strip below which monitoring leads, etc, can enter chamber (9), tubing to pressure monitor (10), and controls for inspiratory and expiratory pressures and time intervals (11)

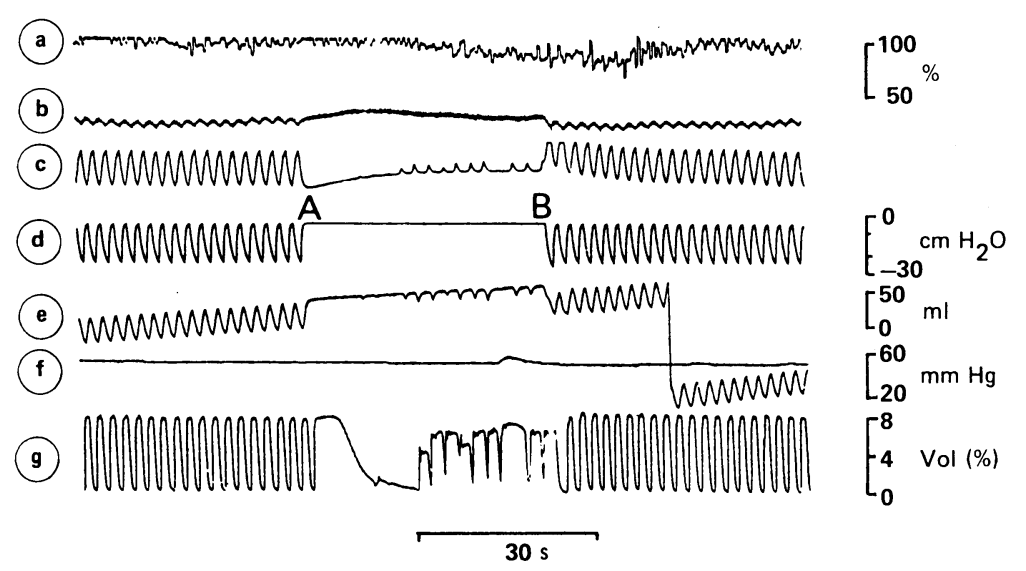

FIG 2-Recording from infant with congenital alveolar hypoventilation syndrome receiving intermitten negative extrathoracic pressure during sleep. (a) Arterial oxygen saturation in beat to beat mode; $(b)$ plethysmographic waveform from which arterial oxygen saturation is derived; (c) inductance plethysmography (inspiration is upward deflection); (d) negative extrathoracic pressure; (e) tidal volume from pneumotachograph; $(f)$ transcutaneous carbon dioxide pressure; and $(g)$ expired carbon dioxide concentration. Between $A$ and $B$ intermittent negative extrathoracic pressure was discontinued: the infant's own breathing efforts were ineffectual

\section{APPARATUS}

The system we used to apply negative extrathoracic pressure was developed over two years (fig 1). The chamber has three different sizes: neonatal $(<4 \mathrm{~kg})$, infant $(3-8 \mathrm{~kg})$, and toddler $(5-20 \mathrm{~kg}$ ) (Horner and Wells, Chelmsford). It has a Perspex lid hinged to one side of an aluminium base with a rubber seal around its edge. The lid has two portholes on each side, which provide access to the patient. While the carer's arms are inside the chamber subatmospheric pressure is maintained by the use of packed cell foam gaskets. Most care procedures can be performed with the infant under subatmospheric pressure, including nursing observations, reapplication of monitoring sensors, physiotherapy, and nappy changes.

The lid of the chamber has a rubber strip along its lower edge that allows monitoring leads and infusion lines to enter the chamber between the base and lid without being constricted and yet still maintain subatmospheric pressure. A recent addition includes a fifth porthole in the end of the chamber lid that allows attachments to be applied and removed without opening the chamber (fig $1(f)$ ). Intra-arterial lines may run into the chamber without any damping of signal content.

The patient's neck lies under an arch in the head end of the chamber lid. An almost complete seal is obtained with a rectangular sheet of latex (thickness $350 \mu \mathrm{m}$ ) (Pentonville Rubber Products, London) in which a hole has been cut with an area about two thirds that of the neck; because of its elasticity it can be stretched over the head of the infant. The side and uppermost edges of the latex are taped (Micropore, $3 \mathrm{M}$ ) to the lid of the chamber while the lower edge is dropped between the base and head section. The head section, which travels in and out on sliding rods, can then be locked into a position that grips the lower edge of the latex. Suction created by the subatmospheric pressure within the chamber causes the latex to adhere and seal both around the arch and over the base of the patient's neck. The elasticity of the latex allows a skin tight seal without circumferential pressure, which might obstruct the upper airway. In addition, an effective neck seal, the patient's comfort, and the avoidance of neck soreness are achieved by the use of double thickness elasticated tubular stockinette (Eesiban, E Sallis, Nottingham). This is cut for the purpose of forming a polo neck vest with a long collar $(5 \mathrm{~cm})$ that lies between the latex and the skin.

Urgent access to the patient can easily be gained by removing the tapes holding the latex on to the lid and releasing two quick release latches that hold the lid down. The lid swings open gradually on gas strut hinges and leaves the patient lying on the base and head section, so that access to the head, trunk, and arms and legs is possible. In addition, intubation or ventilation with a facemask, or both, are facilitated by the removal of one of a pair of head pillows. This allows the patient's neck to be fully extended. Patients breathing spontaneously have additional oxygen supplied within a specially designed head box that fits over the head section or through nasal cannulas (DeVilbiss, Feltham).

The chamber can be tilted with the head uppermost by between $10^{\circ}$ and $20^{\circ}$, and the patient can be nursed prone, supine, or on his or her side and be moved between these positions. The toddler and infant chambers are used on bed or cot bases and the neonatal chambers either on a platform incubator or on a modified incubator base (Horner and Wells, Chelmsford, and Vickers Medical, Basingstoke) which provides the circulation of warmed air, servo controlled to the ambient air temperature within the chamber. This development is particularly important for preterm infants with the respiratory distress syndrome, allowing them to be nursed naked within the chamber.

Subatmospheric pressure was produced by a specially designed unit (DHB Tools, Leamington Spa) incorporating an electrical fan, a safety valve set at -40 $\mathrm{cm} \mathrm{H}_{2} \mathrm{O}$, and a control to adjust the airflow. The first developed suction units provided only a continuous airflow, but the addition of a twin valve system allowed the provision of intermittent larger negative pressures (settings most used were $-18 \mathrm{~cm} \mathrm{H}_{2} \mathrm{O}$ to $-30 \mathrm{~cm} \mathrm{H}_{2} \mathrm{O}$ ) superimposed on to a constant background pressure (usually $-6 \mathrm{~cm} \mathrm{H}_{2} \mathrm{O}$ to $-10 \mathrm{~cm} \mathrm{H}_{2} \mathrm{O}$ ). These can be provided between one and 60 times a minute, with a variable ratio between the inspiratory and expiratory times. Commonly used settings are 20 breaths/min with a ratio of inspiratory to expiratory times of $1: 2$.

The suction unit is connected to the chamber base by a short length of hosing. The pressure developed within the chamber is monitored by means of a purpose built electronic monitor (March Designs, Dunstable) with appropriately set alarm limits. Figure 2 shows the analogue output of the pressure monitor and the 
pressure changes generated within the chamber. In infants receiving intermittent negative pressure ventilation the presence of a constant background negative pressure prevents any large to and fro body motion with each breath.

\section{PROTOCOL FOR USING NEGATIVE EXTRATHORACIC PRESSURE IN RESPIRATORY FAILURE}

All patients were continuously monitored with a validated pulse oximeter ${ }^{13}$ (Nellcor, California) and $\mathrm{SaO}_{2}$ maintained between $92 \%$ and $95 \%$ before term and $95-98 \%$ after term. The inspired oxygen concentrations required to achieve these values were measured while the infant was asleep or quiet with a calibrated oxygen analyser placed by the infant's nose in a head box; if the infant was intubated the oxygen concentration delivered by the ventilator was measured. In addition, depending on the condition of the patient, the following variables were continuously monitored: electrocardiogram, systemic arterial blood pressure, transcutaneous carbon dioxide concentration (Hewlett Packard with Draeger electrode, Uxbridge), and core or peripheral temperature, or both. Arterial or central venous blood samples for measuring $\mathrm{pH}$ and validating transcutaneous carbon dioxide concentration were taken before starting treatment and, depending on the severity of respiratory failure, at variable intervals after starting treatment.

Continuous extrathoracic pressures of between -6 $\mathrm{cm} \mathrm{H}_{2} \mathrm{O}$ and $-10 \mathrm{~cm} \mathrm{H}_{2} \mathrm{O}$ were instituted over several seconds. In the patients already receiving positive airway pressure ventilation the positive peak inspiratory and end expiratory pressures were reduced by the magnitude of negative pressure used. For example, an infant receiving peak inspiratory/end expiratory airway pressures of $+30 / 4 \mathrm{~cm} \mathrm{H}_{2} \mathrm{O}$ would change to $+22 / 0 \mathrm{~cm}$ $\mathrm{H}_{2} \mathrm{O}$ immediately before a constant negative pressure of $-8 \mathrm{~cm} \mathrm{H}_{2} \mathrm{O}$ was applied. In some patients receiving high rate positive airway pressure ventilation it was not always possible to obtain end expiratory pressures of $0 \mathrm{~cm} \mathrm{H}_{2} \mathrm{O}$, values of $+2 \mathrm{~cm} \mathrm{H}_{2} \mathrm{O}$ to $+3 \mathrm{~cm} \mathrm{H}_{2} \mathrm{O}$ being accepted. On starting intermittent negative extrathoracic pressure ventilation in patients who were intubated the positive airway pressure would first be discontinued including any end expiratory pressure, leaving only a constant flow of humidified gases down the endotracheal tube.

In the sickest patients full nursing care and physiotherapy could be performed while negative pressure was maintained. In the less acute cases of respiratory failure the chamber was opened every 4-12 hours for feeding and parental contact. While patients were receiving negative pressure feeds were given through a nasogastric or nasojejunal tube.

To remove the patient from the chamber, when feasible, the negative pressure was tailed off slowly over 5-15 minutes to avoid a sudden fall in lung volume. In infants previously receiving positive pressure ventilation airway pressures were increased appropriately-for example, a patient receiving $15 / 0$ $\mathrm{cm} \mathrm{H}_{2} \mathrm{O}$ would change to $23 / 5 \mathrm{~cm} \mathrm{H}_{2} \mathrm{O}$ on discontinuing negative extrathoracic pressure of $-8 \mathrm{~cm} \mathrm{H}_{2} \mathrm{O}$.

On extubation an infant would usually be kept on either continuous or intermittent negative extrathoracic pressure for a minimum of 24 hours. As the patient's condition improved, increasingly longer times were spent out of the chamber and the pressure was gradually reduced to between $-4 \mathrm{~cm} \mathrm{H}_{2} \mathrm{O}$ and $-6 \mathrm{~cm}$ $\mathrm{H}_{2} \mathrm{O}$. The treatment was discontinued when no change was noted in inspired oxygen concentration with or without negative pressure.

When the patient's condition allowed, parents were encouraged to learn to take part in nursing their child both in and out of the chamber. The parents of patients who were to continue treatment at home had training in the use of the negative pressure respiratory support system and a transcutaneous oxygen monitor (Kontron $821 \mathrm{~S}$, Watford).

\section{Results}

Table II shows the concentrations of inspired oxygen required to achieve the previously defined arterial oxygen saturation before and after applying negative extrathoracic pressure. Table III shows the outcomes in the patients. Seventy five infants required reductions in inspired oxygen concentration two hours after starting treatment (range in reductions $4-50 \%$, median $15 \%$ ) and 74 required reductions after 48 hours (range $2-79 \%$, median $20 \%$ ). The greatest reductions in

TABLE II - Inspired oxygen concentrations (percentages) according to diagnosis in 88 infants before and after start of negative extrathoracic pressure

\begin{tabular}{|c|c|c|c|c|}
\hline \multirow[b]{2}{*}{ Diagnosis } & \multirow{2}{*}{$\begin{array}{l}\text { No of } \\
\text { patients }\end{array}$} & \multicolumn{3}{|c|}{ Inspired oxygen concentration } \\
\hline & & Before & After $2 \mathrm{~h}$ & After $48 \mathrm{~h}$ \\
\hline Bronchopulmonary dysplasia & 47 & & Samuels and Southall ${ }^{1+}$ & \\
\hline Neonatal respiratory distress syndrome & 13 & $\begin{array}{l}40,60,100,100,100,100,60,100,100 \\
100,100,100,100\end{array}$ & $\begin{array}{l}30,55,55,85,85,80,40,70,90 \\
\quad 80,90,87,55\end{array}$ & $\begin{array}{l}21,30,21,100, \star \\
97,95,50,35\end{array}$ \\
\hline Phrenic nerve palsy & 10 & $50,40,40,60,60,40,30,60,60,40$ & $30,30,30,60,50,30,30,60,60,50$ & $30,21,21,60,25,30,21,60,60,40$ \\
\hline Bronchiolitis or asthma & 7 & $30,30,40,30,100,40,100$ & $21,21,21,30,85,30,80$ & $21,21,21,21,95,21,28$ \\
\hline Myopathy & 5 & $30,40,21,40,60$ & $25,30,21,35,40$ & $21,21,21,21,30$ \\
\hline Complications of cardiac surgery & 2 & 50,25 & 50,21 & 50,21 \\
\hline Pneumonitis & 2 & 100,100 & 75,80 & 30,55 \\
\hline Congenital alveolar hypoventilation syndrome & 2 & 30,40 & 30,30 & 30,25 \\
\hline
\end{tabular}

$\star$ Died.

TABLE III-Outcome of treatment with negative extrathoracic pressure in 88 infants

\begin{tabular}{|c|c|c|c|c|c|c|c|}
\hline \multirow[b]{2}{*}{ Diagnosis } & \multirow{2}{*}{$\begin{array}{c}\text { No of } \\
\text { patients }\end{array}$} & \multicolumn{2}{|c|}{$\begin{array}{l}\text { No with reduction in inspired } \\
\text { oxygen concentration after: }\end{array}$} & \multirow{2}{*}{$\begin{array}{l}\text { Proportion of } \\
\text { patients receiving } \\
\text { PAPV who } \\
\text { were extubated }\end{array}$} & \multirow{2}{*}{$\begin{array}{l}\text { No of } \\
\text { deaths }\end{array}$} & \multirow{2}{*}{$\begin{array}{l}\text { No discharged } \\
\text { home } \\
\text { (continuing } \\
\text { treatment) }\end{array}$} & \multirow{2}{*}{$\begin{array}{c}\text { Duration o } \\
\text { treatment } \\
\text { (days) }\end{array}$} \\
\hline & & $2 \mathrm{~h}$ & $48 \mathrm{~h}$ & & & & \\
\hline Bronchopulmonary dysplasia & 47 & 43 & 44 & $8 / 13$ & 20 & $27(3)$ & $2-147$ \\
\hline Neonatal respiratory distress syndrome & 13 & 13 & 9 & $6 / 11$ & 6 & 7 & $2-126$ \\
\hline Phrenic nerve palsy (postoperative) & 10 & 5 & 6 & $7 / 8$ & 3 & $7(1)$ & $3-120$ \\
\hline Bronchiolitis or asthma & 7 & 6 & 7 & $0 / 1$ & 1 & 6 & $2-60$ \\
\hline Myopathy & 5 & 4 & 4 & $2 / 2$ & 3 & $2(1)$ & $13-122$ \\
\hline Complications of cardiac surgery & 2 & 1 & 1 & $2 / 2$ & 1 & 1 & 3 and 8 \\
\hline Pneumonitis & 2 & 2 & 2 & $1 / 1$ & 0 & 2 & 7 and 14 \\
\hline Congenital alveolar hypoventilation syndrome & 2 & 1 & 1 & $2 / 2$ & 0 & $2(1)$ & 28 and 236 \\
\hline Total & 88 & 75 & 74 & $28 / 40$ & 34 & $54(6)$ & $2-236$ \\
\hline
\end{tabular}

PAPV $=$ Positive airway pressure ventilation. 
inspired oxygen were found in infants with the neonatal respiratory distress syndrome and pneumonitis (range $5-45 \%$ (median 20\%) at two hours and 3-79\%, $(35 \%)$ at 48 hours). Results for the infants with bronchopulmonary dysplasia are given elsewhere. ${ }^{1+}$

Of the 14 infants who required no reduction in inspired oxygen concentrations after 48 hours, one underwent plication of the diaphragm (unilateral postoperative phrenic nerve palsy), two were extubated and were given long term intermittent negative pressure ventilation, and 11 died.

Five patients were given intermittent negative extrathoracic pressure: two with bilateral phrenic nerve palsies, one with a metabolic myopathy and two with the congenital alveolar hypoventilation syndrome. In all five cases arterial carbon dioxide pressures were maintained within the normal range for between 12 days and nine months.

In a total of 59 infants carbon dioxide pressures were measured just before and shortly after starting continuous or intermittent negative extrathoracic pressure: 21 showed a reduction in values (range $0 \cdot 6-8.9 \mathrm{kPa}$ (median $2.0 \mathrm{kPa})$ ), 30 showed no change $( \pm 0.5 \mathrm{kPa})$, and eight showed a rise (range $0 \cdot 6-5 \cdot 1 \mathrm{kPa}$, (median $2 \cdot 1$ $\mathrm{kPa})$ ).

Of 40 patients intubated at the start of treatment, extubation was facilitated in 28 and 24 survived. Longer term respiratory support with negative extrathoracic pressure was provided at home in three patients with bronchopulmonary dysplasia, one patient with bilateral phrenic nerve palsies, one with congenital myopathy, and one with the congenital alveolar hypoventilation syndrome.

A total of 34 infants died: 29 from respiratory failure, three from congenital heart disease, one from a cerebral periventricular haemorrhage, and one from necrotising enterocolitis. Four patients who were intubated were able to be extubated before dying of their respiratory disease. In two patients with myopathy treatment with continuous negative pressure was electively discontinued; both subsequently died.

Observation of physiological variables showed that there was a consistent fall in heart and respiratory rates on starting negative extrathoracic pressure. ${ }^{14}$ Systemic blood pressure usually remained unaffected, although there was an increase in some infants receiving positive airway pressure when the peak and end expiratory positive pressures were simultaneously reduced. In the more acutely ill patients the beneficial effects were evident each time negative extrathoracic pressure was given. As respiratory failure resolved the improvements resulting from the treatment remained even when negative pressure was not used, and this dictated the time at which weaning could be pursued.

Chest $x$ ray films showed improvements in the lung fields at varying times after negative extrathoracic pressure was started. Most evident was the resolution of diffuse, soft shadowing. In bronchopulmonary dysplasia hyperlucent and cystic areas did not enlarge. No pneumothorax or other evidence of air leak was seen after starting treatment. Pulmonary vascular markings showed no clearcut changes, although cardiomegaly from right sided heart failure often resolved. Patients seemed to be more settled and showed less respiratory distress after starting respiratory support with negative pressure. Over long periods (up to six months) treatment was often given only overnight and was well tolerated by patients. Parents rapidly learnt to place and care for their children within the chamber.

Potential complications such as fluid retention, intrathoracic air leak, and gastro-oesophageal reflux with aspiration were not experienced. In addition, a pre-existing pneumothorax did not preclude treatment being effective. Upper airway obstruction became evident in only one infant who had bronchopulmonary dysplasia and required tracheostomy for subglottis stenosis after extubation; the tracheostomy still allowed an effective neck seal to be obtained. Neck soreness was not encountered once the most appropriate material to lie between the latex and the neck was found and adopted (see apparatus). Both the neck seal and the modified incubator base overcame problems with cooling in the smallest infants $(<1000 \mathrm{~g})$.

\section{Discussion}

Irrespective of the pathophysiology resulting in respiratory failure (excluding upper airway obstruction) we have shown that the application of negative extrathoracic pressure is a practicable, non-invasive, and effective form of treatment. Negative pressure respiratory support dates back to $1838,,^{12}$ but it fell into disuse because of technical reasons: airway obstruction and soreness from the neck seal, heat loss, and difficulties in access. Access was difficult in earlier versions of the chamber because the patient's head had to be inserted through an iris diaphragm within a rigid aperture. Our system has overcome these problems.

The absence of airway invasion, ease of use by nursing staff and parents, and the recent availability of continuous non-invasive monitoring equipment has made this technique for respiratory support particularly suitable outside intensive care and in non-industrialised countries. In addition, there are physiological reasons why negative extrathoracic pressure lung inflation may be preferable to positive airway pressure: these have been documented in both animal experiments and clinical practice..$^{10.19}$

Continuous negative extrathoracic pressure and continuous positive airway pressure both increase transpleural pressure, thereby helping to splint open small airways and alveoli and re-expand atelectatic regions. Systemic oxygenation depends, however, not only on alveolar expansion and diffusing capacity but also on adequate pulmonary capillary perfusion with matching of perfusion to ventilation. Positive airway pressure reduces cardiac output, ${ }^{1920}$ probably by impairing venous return to the right atrium and by increasing pulmonary vascular resistance. Thus at a certain unpredictable point ${ }^{21}$ increases in positive airway pressure may cause a fall in effective pulmonary blood flow with a resulting increase in ventilationperfusion mismatch ${ }^{22}$ and worsening of hypoxaemia.

Negative extrathoracic pressure, however, increases thoracic volume with less compression of vascular structures and consistently reduces pulmonary vascular resistance, ${ }^{15-18}$ particularly at the pressures used in our patients. In addition, it may dilate pulmonary capillaries and improve ventilation-perfusion matching. This may explain its recently reported value in persistent pulmonary hypertension of the newborn."

Bancalari et al reported the physiological effects of negative extrathoracic pressure in infants with the neonatal respiratory distress syndrome..$^{23}$ They showed a rise in arterial oxygen pressure, a fall in minute ventilation, and no change in arterial carbon dioxide pressure. Our results confirm the effects on gas exchange, and work in progress supports the idea that negative extrathoracic pressure has predominant effects on ventilation-perfusion matching. The changes in carbon dioxide concentrations, however, suggest that an increase in alveolar ventilation does occur in some patients. In addition, our observations that some patients maintain good respiratory function during the day when treated with negative pressure at night suggests that other mechanisms may also be operating. An increase in the patient's functional residual capacity, an increase in lung compliance, support of a compliant chest wall, or a reduction in diaphragmatic fatigue may 
all occur as a result of inflation of the lung under negative pressure.

In hyaline membrane disease constant negative extrathoracic pressure has been compared with nasal positive airway pressure. ${ }^{5}$ Both were effective, although negative pressure produced a more rapid improvement in oxygenation. This may have arisen partly because negative pressure produced a more definitive change in transpleural pressure than a technique relying on the transmission of positive pressure through the nose. In addition, ventilation to perfusion matching may have been enhanced by negative pressure support as discussed above.

The presence of an endotracheal tube is associated with increased bronchial secretions, impairment of ciliary clearance, mucus plugging, and upper airway trauma. It also increases the risk of airway and parenchymal infections. All of these factors may contribute to a continuing need for respiratory support after the acute condition has resolved. Both our experience and that of Mokrin and Bancalari has supported the idea that early initiation of treatment for respiratory failure will reduce the need for intubation. ${ }^{8}$

Non-invasive means of respiratory support wil reduce the incidence of factors that contribute to chronic lung disease, particularly in preterm infants. ${ }^{2+}$ Fanaroff $e t$ al reported that infants with the respiratory distress syndrome treated with negative extrathoracic pressure had less need for positive airway pressure ventilation and a shorter need for supplemental oxygen than infants treated with oxygen alone. ${ }^{+}$Monin et al compared intermittent negative and positive airway pressure ventilation in 115 infants with the respiratory distress syndrome. ${ }^{10}$ There was equal oxygen exposure and no difference in the incidence of patent ductus arteriosus, intracranial haemorrhage, or mortality but a significant reduction in the incidence of pneumothorax and bronchopulmonary dysplasia in those treated with negative pressure.

We cannot state that our system of ventilation is better than other forms of respiratory support. We measured blood gas concentrations and survival, but as we had no control groups interpretation of the results is limited. Our system was developed over two years and appopriate historical controls for the treatment of patients in over 20 centres is not appropriate. We have shown, however, that our system is an effective and safe respiratory support that can be managed by nursing staff and parents in intensive care units, general wards, and at home. Randomised controlled trials in neonatal respiratory failure and in infants still dependent on oxygen or positive airway pressure ventilation, or both, at 28 days of age are now in progress. Their conclusions may have important implications for the future management of respiratory failure in infancy.

We thank Horner and Wells, Huntleigh Technology, Vickers Medical, Vivienne Young of Brunel University, P and M Snowdon, Fisons, Garfield Weston, and the New Moorgate Trust Fund for help in developing this respiratory support system. We thank the nurses who cared for the patients and the doctors who supported us in their management: D Barltrop, M Bommen, J J Bowyer, J M Bridson, $M$ J Bruerton, K Costello, R Dinwiddie, D A Ducker, D J Field, H R Gamsu, A Greenough, P A Hamilton, D Harvey, T J Lissauer, W Lenney, D J Matthew, A C Meeks, N Modi, M C Peard, M L Rigby, R P A Rivers, S A W Salfield, E A Shinebourne, M Silverman, S A Spencer, R M Thomas, B Valman, J O Warner, and A G L Whitelaw. MPS is funded by the Clinical Research Committee of the National Heart and Chest Hospitals (NHCH) and DPS by $\mathrm{NHCH}$ and the Foundation for the Study of Infant Deaths (United Kingdom).

\footnotetext{
1 Woollam CHM. The development of apparatus for intermittent negative pressure respiration 1) 1832-1918. Anaesthesia 1976.31:537-47.

2 Woollam CHM. The development of apparatus for intermittent negative
}

pressure respiration (2) 1919-1976 with special reference to the development and uses of cuirass respirators. Anaesthesia 1976;31:666-85.

3 Bancalari E, Gerhardt T, Monkus E. Simple device for producing continuou negative pressure in infants with IRDS. Pediatrics 1973;52:128-30.

4 Fanaroff AA, Cha CC, Sosa R, Crumrine RS, Klaus MH. Controlled trial of continuous negative external pressure in the treatment of severe respiratory distress syndrome. 7 Pediatr 1973;82:921-8.

5 Alexander G, Gerhardt T, Bancalari E. Hyaline membrane disease. Comparison of continuous negative pressure and nasal positive airway pressure in its treatment. Am $\mathcal{F}$ Dis Child 1979;133:1156-9.

6 Outerbridge EW, Roloff DW, Stern L. Continuous negative pressure in the management of severe respiratory distress syndrome. I Pediatr 1972;81 384-91.

7 Chernick V, Vidyasagar D. Continuous negative chest wall pressure in hyaline membrane disease: one year experience. Pediatrics 1972;49:753-60.

8 Mokrin LD, Bancalari EH. Early versus delayed initiation of continuous negative pressure in infants with hyaline membrane disease. $\mathcal{F}$ Pediat 1975;87:596-600.

9 Silverman WA, Sinclair JC, Gandy GM, Finster M, Baumann WA, Agate FJ A controlled trial of management of respiratory distress syndrome in a body. enclosing respirator. I. Evaluation of safety. Pediatrics 1967;39:740-8.

10 Monin P, Cashore WJ, Hakanson DO, Cowett RM, Oh W. Assisted ventilation in the neonate-comparison between positive and negative respirators. Pediatr Res 1976;10:464.

11 Sills JH, Cvetnic WG, Pietz J. Continuous negative pressure in the treatment of infants with pulmonary hypertension and respiratory failure. f Perinat 1989;9:43-8.

12 Cvetnic WG, Waffarn F, Martin JM. Continuous negative pressure and intermittent mandatory ventilation in the management of pulmonary interstitial emphysema: a preliminary study f P Perinat 1989:9:26-32.

13 Southall DP, Bignall S, Stebbens VA, Alexander JR, Rivers RPA, Lissauer I Pulse oximeter and transcutaneous arterial oxygen measurements in neonatal and paediatric intensive care. Arch Dis Child 1987;62:882-8.

14 Samuels MP, Southall DP. Continuous negative extrathoracic pressure in the treatment of bronchopulmonary dysplasia. Arch Dis Child in press

15 Roos A, Thomas LJ Jr, Nagel EL, Prommas DC. Pulmonary vascular resistance as determined by lung inflation and vascular pressures. f Appl Physiol 1961;16:77-84

16 Thomas LJ Jr, Griffo ZJ, Roos A. Effect of negative pressure inflation of the lung on pulmonary vascular resistance. $\mathcal{F}$ Appl Physiol 1961;16:451-6.

17 Kira S, Hukushima Y. Effect of negative pressure inflation on pulmonary vascular flow. 7 Appl Physiol 1968;25:42-7.

18 Burton AC, Patel DJ. Effect on pulmonary vascular resistance of inflation of the rabbit lungs. $\mathcal{F}$ Appl Physiol 1958;12:239-46.

19 Krumpe PE, Zidulka A, Urbanetti J, Anthonisen NR. Comparison of the effects of continuous negative external chest pressure and positive endexpiratory pressure on cardiac index in dogs. Am Rev Respir Dis 1977;115: expirat.

20 Tyler DC. Positive end-expiratory pressure: a review. Crit Care Med 1983;11:300-8

21 Witte MK, Galli SA, Chatburn RL, Blumer JL. Optimal positive end expiratory pressure therapy in infants and children with acute respiratory failure. Pediatr Res 1988;24:217-21

22 Nelson RM, Egan EA, Eitzman DV. Increased hypoxemia in neonates secondary to the use of continuous positive airway pressure. $f$ Pediat 1977;91:87-91.

23 Bancalari E, Garcia OL, Jesse MJ. Effects of continuous negative pressure on lung mechanics in idiopathic respiratory distress syndrome. Pediatric 1973;51:485-93.

24 Bancalari E, Stocker JT, eds. Bronchopulmonary dysplasia. Washington, DC: Hemisphere, 1988.

(Accepted 27 September 1989)

\section{ONE HUNDRED YEARS AGO}

The following authenticated facts throw some light upon one of the so-called cases of "faith healing" which has been promulgated by the press urbi et orbi. Richard Rummery, a patient who was "blind and bedridden," and cured by faith, has, we have ascertained, been suffering for some time from rheumatoid neuralgia, accompanied by depression and attacks of a hysterical character. He has also a post-polar cataract in the right eye. On returning from Bath he suffered from conjunctivitis in both eyes, with intense photophobia, but was much better both in general health and as regards the condition of the sound eye when the faithhealing incident occurred. Excepting that he gets up and out of doors, which, no doubt, he could have done previously had he been so minded, his condition is practically unchanged. We are informed that he has never been in any real danger. (British Medical fournal 1889;ii:86)

\section{Correction}

\section{Recovery after subarachnoid haemorrhage}

Miss P McKenna and others would like to clarify the data in table II of their article (19 August, p 485). The categories given for reduced quality of life are not mutually exclusive, and the number of patients with a reduced quality of life attributable to illness is in fact, 12 (seven of whom complained only of having less energy) and not 21 as suggested by the table. 